\title{
Merging galaxies in compact groups: analytical theory
}

\author{
Victor M. Kontorovich \\ Institute of Radio Astronomy, 4 Krasnoznamennaya St., Kharkov \\ 310002, Ukraine
}

\begin{abstract}
The predominance of minor mergers, i.e. the non-locality of mergings, results in a mass function slope ("Shechter" index) intermediate between a power-law index of a spectrum with constant mass flux and that of constant flux of the number of massive galaxies. The index proves to be close to that of the faint end of the galaxy luminosity function.
\end{abstract}

This paper is concerned with the problem of the steepness of the galaxy luminosity function (LF) at its faint end (see e.g. Marzke et al. 1998 and references therein). In particular, it deals with the intermediate asymptotics (IA) of the galaxy mass function (MF) arising from galaxy mergers and with its physical interpretation.

Before examining real galaxy interactions we recall that the Smoluchowski Equation (SE), which describes the merging process,

$$
\frac{\partial f(m, t)}{\partial t}=\int d x\left[U_{m \mid 12} \delta_{m} f_{1} f_{2}-\text { cycle }- \text { bicycle }\right],
$$

permits two exact solutions (we do not consider here the case of $U=$ const) describing the MF evolution from its initial state localized on small masses (Voloshchuck, 1984; Ernst, 1986). Here MF $f_{1} \equiv f\left(m_{1}, t\right)$ and so on, and $\delta_{m} \equiv \delta\left(m-m_{1}-m_{2}\right)$ is Dirac's $\delta$-function. In the second and third terms we have $\delta\left(m_{2}-m-m_{1}\right)$ and $\delta\left(m_{1}-m_{2}-m\right)$ respectively.

For a merging probability proportional to the product of colliding masses $U_{m \mid 12}=c m_{1} m_{2}$ the power-law index of IA is $s_{1}=-5 / 2$. Expressed through the uniformity power of the merger coefficient $u\left(U(a m)=a^{u} U(m)\right)$ it is $s_{1}=$ $-(u+3) / 2(u=2)$. As is well known (see also below) such an index co6rresponds to a constant mass flux in the spectrum. In the case of $U=c\left(m_{1}+m_{2}\right)$ the power of IA is $s_{0}=-3 / 2$ or $s_{0}=-(u+2) / 2(u=1)$. The latter corresponds to the constant flux of the number of massive objects. Though prima facie such a conservation law fails with the mergers it is realized in the form of an approximate integral in the case of predominant interaction of large objects with the small ones. Such a "non-local" situation fits the last solution. The condition of locality, i.e. that of convergence, requires that the solution is of the form $u_{2}-u_{1}<1$ (Vinokurov and Kats, 1980), where the indexes $u_{1}$ and $u_{2}$ are defined by the expression for $U$ provided the masses differ strongly $U \propto$ $m_{1}^{u_{1}} m_{2}^{u_{2}}\left(m_{1} \ll m_{2}\right)$. Obviously, in the first case $\left(u_{1}=u_{2}=1\right)$ the locality criterion is fulfilled and in the second the marginal case occurs $\left(u_{1}=0, u_{2}=1\right)$. Namely, the galaxy interaction with the most dominant scales prevails and, thus, the "number of particles' conservation law is realized. 
For gravitational galaxy interaction the cross-section of mergers is usually taken as a product of co-factors, which describe, respectively, the geometrical cross-section, gravitational focusing and conditional merging probability for the head-on collision of galaxies (see references in Kontorovich, 1994):

$$
\sigma=\pi r^{2}(1+\gamma) \varphi(\gamma), \quad \gamma \equiv v_{g}^{2} / v^{2}, \quad v_{g}^{2}=2 G m / r, m=m_{1}+m_{2}, r=r_{1}+r_{2}
$$

The homogeneity index differs for "large" and "small" masses. In the case of compact galaxy groups the region of "large" masses is more relevant due to small velocity dispersion (Dressler, 1984). We will focus on this particular region, by allowing a small mass region to contract to zero. This scheme can be described more accurately by the following formalism. On the assumption that $\varphi$ decreases as the square of the relative velocity we can use the form $\varphi=(1+1 / \gamma)^{-1}$. The resulting cross-section will be a uniform function over the whole mass-changing interval: $\sigma=\pi r^{2} \gamma$. By averaging over velocities we come to the merging coefficient $U=\left\langle\sigma v>\right.$ in the form $U \propto\left(m_{1}+m_{2}\right)\left(m_{1}^{\beta}+m_{2}^{\beta}\right)$, where the radius-mass dependence is chosen as $r \propto m^{\beta}$. Below we employ only the fact that $U$ is the uniform function of masses with $u=1+\beta, u_{1}=0, u_{2}=u{ }^{1}$ For $u>1$ the evolution of MF has an explosive character and a quasi power asymptotic is established in a wide mass interval between the region of initial mass localization $m \sim m_{*}$ and the merger front which goes to infinite mass in a finite time (Cavaliere et al, 1992; Kontorovich et al, 1992).

Both the numerical solution of SE and modelling by Monte Carlo simulations show that "Shechter's" index of IA, $\alpha$ (Binggeli et al, 1988) lies between $(u+2) / 2$ and $(u+3) / 2$ (see for example Kontorovich et al, 1995, Fig. 2b; Krivitsky and Kontorovich, 1997). In order to understand what this means consider the symmetry properties of the collision integral of SE in the case of exact uniformity of $U: U_{a m_{1} a m_{2} \mid a m}=a^{u} U_{m_{1} m_{2} \mid m}$. To utilize the uniformity of $U$ we must change simultaneously the scale of all three arguments $m_{1}, m_{2}$ and $m$. But, as one of them $(m)$ is fixed in SE, from the continuous group transformation only two discrete transformations remain (except a trivial one): $G_{1}$ transforming $m_{1} \rightarrow m$ and $G_{2}$ transforming $m_{2} \rightarrow m$ :

$$
\begin{aligned}
& G_{1}=m_{1} \rightarrow \frac{m}{m_{1}} m_{2}, \quad m \rightarrow \frac{m}{m_{1}} m_{1}, \quad m_{2} \rightarrow \frac{m}{m_{1}} m \\
& G_{2}=m_{1} \rightarrow \frac{m}{m_{2}} m, \quad m \rightarrow \frac{m}{m_{2}} m_{2}, \quad m_{2} \rightarrow \frac{m}{m_{2}} m_{1} .
\end{aligned}
$$

These Zakharov transformations are considered as some change of variables $m_{1}, m_{2}$ with the fixed mass $m$ conditions. They form a symmetry group of SE (Kats and Kontorovich, 1977). For these conformal transformations the integrating paths tending to infinity in the second and third terms in (1) convert into the integrating path with the finite mass variation in the first term of $\mathrm{SE}$.

\footnotetext{
${ }^{1}$ The explicit form of $U$ allows us to obtain the formal power-law solution for $U=\left(m_{1}^{\eta}+\right.$ $\left.m_{2}^{\eta}\right)\left(m_{1}^{\beta}+m_{2}^{\beta}\right)$, but in a parameter region irrelevant for our purposes: $\eta, \beta<1$ (Pechernikova, 1987). In this case it is very essential that $\eta=1 \quad(\beta=1 / 3$ to $1 / 2)$.
} 
In the issue (using also $x \rightarrow m-x$ symmetry) SE results in the form of:

$$
\begin{gathered}
\frac{\partial f(m, t)}{\partial t}=2 \int_{0}^{m / 2} d m_{1} d m_{2} U_{m \mid x, m-x}\{\} \\
\{\}=f(m-x) f(x)-\left(\frac{m}{x}\right)^{2+u} f\left(\frac{m}{x}(m-x)\right) f(m)-\left(\frac{m}{m-x}\right)^{2+u} f\left(\frac{m x}{m-x}\right) f(m) .
\end{gathered}
$$

If in addition a power-law character of the solution is assumed, then \{\} in SE is reduced to (Zakharov et al, 1992; Vinokurov et al, 1985):

$$
\{\}=f(m-x) f(x)<1-\left(\frac{m}{x}\right)^{\nu}-\left(\frac{m}{m-x}\right)^{\nu}>, \quad \nu=2+u+2 s .
$$

In the stationary case we get the exact power solution $f \propto m^{s_{1}}, \nu=-1$, which corresponds to the constant mass flux $P$ on the spectrum $f_{P}=c_{1} P^{1 / 2} m^{s_{1}}$. This can be easily proved by using the definition of mass flux, thus finding the normalization factor and the flux sign (Vinokurov and Kats, 1980). The obtained formal solution, however, is non-local: the integrals diverge at small masses, which thus must on the main contribute.

Now consider the MF decreasing steeper than the power on the largest masses. With this condition the second term in (2) vanishes in the case of essential contribution of small masses due to non-locality. An approximate power solution arises that corresponds to conservation of the number of massive galaxies (if their interaction with the small-mass ones prevails):

$$
\{\}=f(m-x) f(x)<1-\left(\frac{m}{m-x}\right)^{\nu}>; f \propto m^{s_{0}}, \quad \nu=0 .
$$

Using the definition of particle flux $Q$ we can normalize this distribution too: $f_{Q}=c_{0} Q^{1 / 2} m^{s_{0}}$ (cf. Kats, 1976). With the two concurrent fluxes it is easy to find analogous solutions, when one of the fluxes is smaller than the other. The value of this ratio depends on the mass: $m Q / P$. We obtain a spectrum with a break at $P / m_{b r} Q \sim 1$ and which goes over to the single-flux distributions at either end. However, our whole case is non-local in principle, and even the additional conservation law is connected with this non-locality (cf. Balk et al, 1990). Thus, we have to proceed to the differential description, primarily to account for the interaction with multiple dwarf galaxies. With the original SE form (1) this is difficult to do in view of the divergence at small $m_{1}$ and $m_{2}$, as well as at infinite mass. After Zakharov transformations we have only one singular point $\left(m_{1}=0\right)$. Expanding near to which gives us the equation:

$$
\begin{gathered}
\frac{\partial f(M, t)}{\partial \tau}=-A\left(\frac{\partial f(m)}{\partial m}+\frac{2+u}{m} f(m)\right)-f(m) \int_{0} d x\left(\frac{m}{x}\right)^{2+u} f\left(\frac{m^{2}}{x}\right)- \\
-\frac{f(m)}{m} \int_{0} d x x^{2} \frac{\partial f(x)}{\partial x} ; \quad A=\int_{0} d x x f(x), \quad\left(\tau=t \cdot 2 U_{m \mid 0 m}\right) .
\end{gathered}
$$

The afore-mentioned compensation occurs here automatically. For the pure power-law distribution we obtain the equation: 


$$
-\frac{\partial \Phi}{\partial \tau}=A \frac{\partial \Phi}{\partial m}+B \Phi^{2}, \quad \Phi \equiv m^{\mu} f(m, t), \quad B=\int_{0} d x / x^{\mu}, \quad \mu=2+u+s .
$$

The formal solution of (6) $1 / \Phi=C m+D \quad(C=B / A)$ gives us two asymptotics. One corresponds to $s_{1}$, the other to $s_{0}$ :

$$
1 / \Phi=C m \quad\left(f \propto m^{s_{1}}, C m \gg D\right) ; \quad 1 / \Phi=D \quad\left(f \propto m^{s_{0}}, C m \ll D\right) .
$$

Thus we can affirm (see Krivitsky and Kontorovich, 1997) that if the SE solution is approximated by a power-law function its index lies in the range $-2-\beta / 2<s<-3 / 2-\beta / 2$.

The resulting index can be somewhat smaller or larger than -2 and close to Shechter's index $\alpha \simeq-1.71 \pm 0.5$ (Brunello et al. 1998, for field galaxies), $\alpha \simeq-2.2 \pm 0.3$ (de Propris et al. 1995, in rich clusters)) at the faint end of the LF. If $s<-2$ the major part of the mass of the system is concentrated in the small members of the group.

The non-stationary problem (analogous to Kontorovich et al. 1992 for the case $u=2$ ) leads to the explosive evolution of the MF.

Thus, using only purely analytical methods, we confirm the results of numerical computations of the MF for merging and establishe that the index obtained corresponds to a spectrum where the fluxes of both the mass and the number of (massive) galaxies is in accordance with the exact and approximate conservation laws for the conditions of non-locality of the distributions.

\section{References}

Balk, A. M., Zakharov, V. E., \& Nazarenko, S. V., 1990, Zhurn Exp Teor Fiz (Sov.Phys.JETP), 98, 446

Binggeli, B., Sandage, A., \& Tammann,G. A. ARA\&A, 1988, 26, 509

Brunello, B., Saracco, P., Garilli, B., et al., in Observational Cosmology: The Development of Galaxy Systems, G. Giuricin, M. Mezetti \& P. Salucci, ASP Conf Ser 176, San Francisco, 276

Cavaliere, A., Colofrancesco, S., \& Menci, N., 1992, ApJ, 392, 41

Cavaliere, A., \& Menci, N., 1997, ApJ, 480, 132

Dressler, A. 1984, ARA\&A, 22, 185

Ernst, M. H. in Fractals in Physics/ Eds. L. Pietronero and E. Tosatti, NorthHolland, Amsterdam, 1986, 399

Kats, A. V., 1976, Sov.Phys.JETP, 71, 2104

Kats, A. V., \& Kontorovich, V. M., 1977, Physica A, 86, 471

Kontorovich, V. M., Krivitsky, D. S., \& Kats, A. V., 1995, Physica D, 87, 290

Kontorovich, V. M., Kats, A. V., \& Krivitsky D. S., 1992, JETP Lett., 55, 1

Krivitsky, D. S., \& Kontorovich, V. M., 1997, A\&A, 327, 921

Marzke, R. O., da Costa, L. N., Pellegrini, P. S., et al., 1998, astro-ph/9805218 Pechernikova, G. V., 1987, Kinematika i Fizika Nebesnykh Tel, 3, No 5, 85 
de Propris, R., Pritchet, C. J., Harris, W. E., et al., 1995, ApJ, 450, 534 Vinokurov, L. I., Kats, A. V., \& Kontorovich, V. M., 1985, J.Stat.Phys., 38, 217 Vinokurov, L. I., \& Kats, A. V., 1980, Fizika Atmosfery i Okeana 16, 601 Voloshchuk, V. M., 1984, Kinetic Theory of Coagulation. GMI, Leningrad Zakharov, V. E., L'vov, V. S., \& Falkovich, G., 1992, Kolmogorov spectra of turbulence. Springer, Heidelberg 